\title{
3.13 NUTRITION AND THE HOSPITALIZED PATIENT
}

Optimal nutrition in the hospital can facilitate better patient outcomes. Malnutrition in hospitalized patients can lead to poor wound healing, impaired immune function resulting in infectious complications, increased hospital length of stay, increased risk of readmission, and overall increased morbidity and mortality. ${ }^{1,2}$ Malnutrition is reported in up to $50 \%$ of hospitalized patients. Although early screening for nutritional risk allows for appropriate intervention in the hospital setting as well as planning for appropriate home services and follow-up for outpatient nutritional care, malnutrition is underrecognized and undertreated. ${ }^{3}$ In malnourished patients, nutritional intervention has been shown to reduce clinical complications, length of stay, readmission rates, and mortality. ${ }^{4}$ Hospitalists use a multidisciplinary approach to evaluate and address the nutritional needs of hospitalized patients. Hospitalists lead, coordinate, or participate in multidisciplinary initiatives to improve the nutritional status of hospitalized patients.

\section{KNOWLEDGE}

Hospitalists should be able to:

- Describe methods of screening for malnutrition.

- Describe the consequences of malnutrition on bodily function, illness, and outcomes.

- Explain when a nutrition evaluation by a registered dietitian is required.

- Differentiate among various modified diets and nutritional supplements and explain the indications for each.

- Explain the indications and contraindications for enteral nutrition.

- Describe the indications for parenteral nutrition.

- Describe potential complications associated with enteral and parenteral nutrition.

- Recognize that specialized nutritional supplementation may be required in certain patient populations, which include patients with extensive wounds or increased catabolic needs.

- Explain the risk factors for and the clinical features of the refeeding syndrome.

\section{SKILLS}

Hospitalists should be able to:

- Use objective criteria, including history, physical examination findings, and laboratory results, to diagnose and categorize the severity of malnutrition and identify patients who are at increased risk.
- Identify the symptoms or signs of medical conditions that are associated with or secondary to malnutrition and formulate an evidence-based treatment plan.

- Implement individualized modified diets and/or nutritional supplements, which may include total parenteral nutrition, on the basis of the patient's medical condition.

- Treat electrolyte abnormalities associated with the refeeding syndrome.

- Monitor electrolytes as indicated in the setting of enteral and/or parenteral nutritional support.

- Consult a nutrition specialist for a comprehensive nutritional evaluation when indicated.

- Coordinate follow-up nutrition care as part of discharge plans for those patients requiring nutritional support.

- Lead, coordinate, and/or participate in initiatives to improve awareness and documentation efforts that appropriately categorize the patient with malnutrition and determine the impact this may have on risk-adjusted mortality and value-based purchasing.

- Lead, coordinate, and/or participate in multidisciplinary initiatives to optimize resource use.

- Lead, coordinate, and/or participate in the development of care pathways for patients requiring enteral or parenteral nutrition.

- Lead, coordinate, and/or participate in efforts to develop strategies to minimize institution complication rates.

\section{ATTITUDES}

Hospitalists should be able to:

- Recognize the importance of adequate nutrition in hospitalized patients.

- Work collaboratively with clinical nutrition staff, which may include nursing, pharmacists, and dieticians, to implement the nutrition care plan.

- Engage in a team approach for early discharge planning for patients requiring home parenteral or enteral nutrition.

\section{References}

1. Allaudeen N, Vidyarthi A, Maselli J, Auerbach A. Redefining readmission risk factors for general medicine patients. J Hosp Med. 2011;6(2):54-60.

2. Kassin MT, Owen RM, Perez SD, Leeds I, Cox JC, Schnier K, et al. Risk factors for 30-day hospital readmission among general surgery patients. J Am Coll Surg. 2012;215(3):322-330.

3. Mitchell MA, Duerksen DR, Rahman A. Are housestaff identifying malnourished hospitalized medicine patients? Appl Physiol Nutr Metab. 2014;39(10):1192-1195.

4. Tappenden KA, Quatrara B, Parkhurst ML, Malone AM, Fanjiang G, Ziegler TR. Critical role of nutrition in improving quality of care: an interdisciplinary call to action to address adult hospital malnutrition. J Acad Nutr Diet. 2013;113(9):1219-1237. 